\title{
Statistical Theory of Unimolecular Reactions and Intramolecular Dynamics
}

\section{R. A. MARCUS}

Noyes Laboratory of Chemical Physics, California Institute of Technology, Pasadena, CA 91125

In the present lecture we review experimental and theoretical developments in the field of intramolecular dynamics during the past sixty years. In a concluding section we consider possible implications for intramolecular laser selective chemistry.

Unimolecular processes in the gas phase have been the subject of considerable interest for more than sixty years. We review some of this history in the present lecture, and conclude with a discussion of some factors which may lead to an intramolecular laser-selective chemistry.

The first historical period which we cover is the period 1919-1952. Around 1920 the decomposition of $\mathrm{N}_{2} \mathrm{O}_{5}$ provided an example of a unimolecular reaction. Unimolecular reactions were a puzzle, in that a collisional activation mechanism should cause the reaction to be second order (rate proportional to the square of the concentration) whereas the observed rate was proportional to the first power. A radiation hypothesis was considered, but discarded on several grounds. Then in 1922 Lindemann postulated a mechanism for reaction of a gas $A$,

$$
\begin{aligned}
& A+A \underset{k_{2}}{\stackrel{k_{1}}{\rightleftharpoons}} A^{*}+A \\
& A^{*} \underset{k_{3}}{\rightleftharpoons} \text { products }
\end{aligned}
$$

in which an energetic molecule $A^{*}$ was formed, and could either form products or be deactivated, as in (1) and (2). A steady-state analysis 
gives

$$
-d A / d t=k_{1} k_{3}(A)^{2} /\left[k_{2}(A)+k_{3}\right]
$$

At high pressures, the rate is seen to be proportional to the first power of $(A)$, even though the activation step (1) is second order. Furthermore, Eq. (3) also makes the interesting prediction that at low $(A)$ the rate is given by $k_{1}(A)^{2}$.

The mechanism (1)-(2) was analyzed further by Hinshelwood, using a statistical theory for the various rate constants in (3). He treated $k_{3}$ to be independent of the vibrational energy of $A$. Theories were also given in the mid-1920s by Rice and Ramsperger and by Kassel. They used a more elaborate statistical theory, which predicted an almost exponential dependence of $k_{3}$ on the vibrational energy of $A$. For example, for a molecule of $s$ participating classical vibrations, with an energy $E$ and a threshold energy for reaction $E_{0}, k_{3}$ was given by

$$
k_{3}=\nu\left(\frac{E-E_{0}}{E}\right)^{s-1}
$$

These treatments utilized Eq. (3), but contained the right-hand side of (3) as an integrand, the integration being over all energies of $A^{*}$. The theory gave theoretical expressions for each of the rate constants in terms of some adjustable parameters (principally two, $s$ and $\nu$, plus $E_{0}$ largely determined from the activation energy), and has become known in the literature as RRK theory.

In the ensuing 1920s and 1930s numerous organic reactions were studied in the gas phase, e.g., decomposition of acetaldehyde to $\mathrm{CH}_{4}$ and $\mathrm{CO}$, and interpreted in the light of these statistical theories. But, then the bubble burst! Each of these reactions was found to proceed via more complex chemical mechanisms involving free radicals. Free radicals became the focus of attention and the theory was left, essentially, without an experimental candidate-even the $\mathrm{N}_{2} \mathrm{O}_{5}$ decomposition was found some decades later to involve a free radical mechanism!

In a quite different area, for photochemically-induced predissociation, the theory of radiationless transitions was developed in the late 1920 s by O. K. Rice and by Wentzel, but this field was rather apart from that of thermally-induced unimolecular reactions.

The transition state theory of chemical reactions was developed in 1935 in its commonly used form by Henry Eyring and by Evans and 
Michael Polanyi. Significant contributions were also made by others (e.g., Wigner, O. K. Rice and Gershinowitz). The theory was devised for reactions at a given temperature. In that form it was not suited for the type of nonequilibrium behavior embodied in Eqs. (1) to (3), and was not applied to the latter. A statistical theory of unimolecular reactions was described by Landau in the middle 1930s, in which he assumed that the molecule could be treated as a large heat bath. In this sense its statistical mechanics was more approximate than RRK, but it did have the virtue of using actual molecular heat capacities in its calculations. It was, in effect, a canonical-type theory applied to a microcanonical problem. Unlike RRK, it has rarely been applied to any data.

In the early and middle 1950 s N. B. Slater developed a theory, elaborating substantially on an early work by Pelzer and Wigner. Slater treated the molecule as a collection of classical mechanical harmonic oscillators (normal modes). Using a statistical-type theory, subject to this constraint, he calculated the mean rate at which some bond length exceeded a preassigned finite value ("truncated harmonic oscillator" model). This rate was regarded as the rate of the dissociation step, Eq. (2). In Slater's theory, in contrast with RRK, there were $N$ ("hidden") constants of the motion for a molecule of $N$ vibrations (the $N$ individual harmonic oscillator energies, for example). In RRK theory only the total vibrational energy was constant.

In 1951-1952 we published several papers on what has since become known as RRKM theory. In this approach transition state theory was reformulated on a microcanonical instead of a canonical basis. The theory assumed, as in RRK, that the anharmonicity of the vibrations was sufficient to make the energy and (angular momentum) the only constants of motion of interest. The treatment was a quantum one and related the kinetic behavior in (1) and (2) to molecular properties of the reacting molecule, such as the potential energy surface for the reaction. For example, the RRKM value for $k_{3}$ in Eq. (2) is given by

$$
k_{3}=\frac{N^{\ddagger}(E)}{h \rho(E)}
$$

where $N^{\ddagger}(E)$ is the number of quantum states of the transition state having an energy less than or equal to $E$ (this $E$ includes that present 
as potential energy), and $\rho(E)$ is the density of quantum states of the reactant $A^{*}$ at the same total energy. $k_{3}$ described by (5) depends also on $J$, the total angular momentum (notation suppressed), since both $N^{\ddagger}$ and $\rho$ can depend on $J$.

The period 1952-1960 was a relatively quiet one for unimolecular reactions. There were some applications of Slater's theory, but later experiments revealed drawbacks of the approximations involved. In the field of mass spectra Rosenstock et al. had developed in 1952 a statistical theory for unimolecular decomposition of ions. The theory, known as the quasiequilibrium theory, was classical rather than quantum and was extensively applied to the data. It developed that the appearance potentials were inconsistent with the rate constants for the molecules involved, because of the approximation of using classical rather than quantum vibrations. Wolfsberg pointed this out in 1962, to explain the discrepancies which had appeared, and applied RRKM theory to the problem. This period of 1952-1960 was not, however, a quiet one for the field of reaction kinetics as a whole. There were the exciting developments in studies of fast reactions-the pioneering work of Norrish and Porter in flash photolysis and of spectroscopic observation of short-lived gas phase free radicals and that of Eigen and co-workers on fast reactions in solution.

The major set of experiments which were to have a profound effect on the field of unimolecular reactions, on the application of RRKM theory, and on its subsequent application to new experiments came during the next ten year period 1960-1970, principally by Rabinovitch and co-workers. These studies included unimolecular isomerizations, such as reaction (6), as well as the production of vibrationally-excited $\left({ }^{*}\right)$ radicals and molecules by the chemical activation technique by addition of an atom, such as $H, D$, or $R$ to an olefin (reaction 7), by addition of a $\mathrm{CH}_{2}$ group (reaction 8), or by a $\mathrm{CH}_{2}$ or $\mathrm{O}$ insertion reaction.

$$
\begin{aligned}
\mathrm{CH}_{3} \mathrm{NC} & \longrightarrow \mathrm{CH}_{3} \mathrm{CN} \\
\mathrm{H}+\mathrm{CH}_{3} \mathrm{CH}_{2} \mathrm{CH}=\mathrm{CH}_{2} & \longrightarrow \mathrm{CH}_{3} \mathrm{CH}_{2} \mathrm{CH}_{2} \mathrm{CH}_{2}^{*} \\
& \longrightarrow \mathrm{CH}_{3} \mathrm{CH}_{2}+\mathrm{CH}_{2}=\mathrm{CH}_{2} \\
\mathrm{CH}_{2}+\mathrm{RCH}=\mathrm{CH}_{2} & \longrightarrow \mathrm{R}-\mathrm{CH}-\mathrm{CH}_{2}^{*} \\
& \longrightarrow \mathrm{CCH}=\mathrm{CH}_{2} \mathrm{CH}_{3}
\end{aligned}
$$


With this chemical type of excitation molecules of a relatively narrow vibrational energy range could be prepared, in contrast with the broader energy range found in thermally-excited molecules. Further, by suitable use of different reactions, the effect of varying the excess vibrational energy could be explored. Systematic studies of series of alkyl radicals were also investigated.

Detailed comparisons of the data with RRKM theory were made, and provided excellent support for the latter. Rabinovitch was also able to estimate from his experiments the time for vibrational energy randomization ( $~ 1 \mathrm{ps}$ for example), e.g., the time for the energy to "randomize" between the two rings in (9),

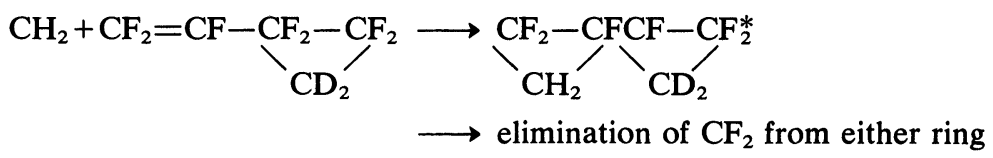

as well as in other quite different systems.

In the 1960s there was also an extensive application and extension of radiationless transition theory to various optically induced processes, such as internal conversion and intersystem crossing. See for example, the important work of Robinson and Frosch. The theory contained an interesting classification of the behavior of small molecules based on their being in the "small," "intermediate" (sparse structure) and "statistical" ("continuum" of final states) regimes, and served to provide a very useful basis for understanding the types of decay-exponential or nonexponential, the effect (in more recent years) of the initial state preparation, and various other aspects.

The next period, 1970-present, saw the introduction of many new experimental techniques on one hand, and the beginnings of a more detailed theoretical investigation of the intramolecular dynanisiss of small anharmonic systems on the other. Among these new techniques was the use of crossed molecular beams to form molecular complexes, whose dissociation behavior could then be studied, notably by Herschbach and by Lee and their collaborators. One can show that if the transition state in the exit channel of the reaction is "loose," e.g., in the transition state of (11)

$$
\begin{aligned}
\mathrm{Cl}+\mathrm{R}_{2} \mathrm{C}=\mathrm{CH}_{2} \mathrm{CHBr} & \longrightarrow \mathrm{R}_{2} \mathrm{ClCCH}_{2} \mathrm{Br} \\
\mathrm{R}_{2} \mathrm{ClC}^{\mathrm{C}} \mathrm{HCH}_{2} \mathrm{Br} & \longrightarrow \mathrm{R}_{2} \mathrm{ClCH}=\mathrm{CH}_{2}+\mathrm{Br}
\end{aligned}
$$


the translational energy distribution of the products should reflect those of the transition state, when angular momentum restrictions are taken into account. Thus, measurement of the former then reflects that of the latter, which in turn can be computed from RRKM theory when the transient molecule behaves as though its energy has "randomized." This agreement with RRKM has been confirmed in experiments, provided the lifetime of the intermediate, e.g., of the $\mathrm{H}_{2} \mathrm{ClCHCH} \mathrm{H}_{2} \mathrm{Br}$, is not too short. In the particular example (10) cited, when $\mathrm{R}=\mathrm{H}$, it appears to be too short ( $\sim 1$ ps perhaps). We have stressed elsewhere the importance of selecting systems without exit channel effects (e.g., $\mathrm{CH}_{3}$ elimination or $\mathrm{H}$ elimination have such effects). The method provided an interesting new approach to learn indirectly about the energy distribution in the transition state when exit channel effects are absent. The vibrational energy distribution of the products has also been examined (McDonald) and similar remarks apply.

Another technique which was introduced in the 1970s was infrared multiphoton dissociation, e.g., the absorption of many infrared quanta followed by dissociation (or isomerization), as in the reaction studied by Stephenson et al.

$$
\mathrm{CF}_{2} \mathrm{CFCl}^{n} \stackrel{h \nu_{\text {ir }}}{\longrightarrow} \mathrm{CF}_{2}+\mathrm{CFCl}
$$

and in many other reactions, such as those studied in molecular beams by Lee and collaborators. Reactions such as

$$
\mathrm{SF}_{6}^{n} \stackrel{h \nu_{i r}}{\longrightarrow} \mathrm{SF}_{5}+\mathrm{F}
$$

have been studied in very short time experiments by Yablonivitch and Bloembergen.

In general, these infrared multiphoton results are thus far consistent with a model in which the lowest vibrational states are "regular" (discussed later) and the higher ones are "statistical" (RRKM). In the case of reaction (12) the vibrational energy distribution of $\mathrm{CF}_{2}$ and the translational energy distribution of the products supported the applicability of statistical theory. Another interesting type of intrared multiphoton study was by Setser et al. on a reaction involving a "branching ratio."

$$
\begin{aligned}
\mathrm{CH}_{2} \mathrm{FCH}_{2} \mathrm{Br}^{n} & \stackrel{h v_{\text {ir }}}{\longrightarrow} \mathrm{CH}_{2} \mathrm{FCH}_{2} \mathrm{Br}^{*} \\
\mathrm{CH}_{2} \mathrm{FCH}_{2} \mathrm{Br}^{*} & \longrightarrow \mathrm{CH}_{2}=\mathrm{CHBr}^{*} \mathrm{HF} \\
\text { or } & \longrightarrow \mathrm{CHF}=\mathrm{CH}_{2}+\mathrm{HBr}
\end{aligned}
$$


where the bond initially excited in the infrared was the CF bond. Measurements of the ratio $\mathrm{HF} / \mathrm{HBr}$ were consistent with $\mathrm{RRKM}$ theory, rather than with a theory in which the selective CF excitation led to selective $\mathrm{HF}$ formation.

Another technique (late 1970s) involves the photodissociation of van der Waals' complexes (Levy et al. and other groups) such as

$$
\begin{aligned}
\mathrm{I}_{2} \mathrm{He} & \stackrel{h \nu}{\longrightarrow} \mathrm{I}_{2}(v) \mathrm{He} \\
\mathrm{I}_{2}(v) \mathrm{He} & \longrightarrow \mathrm{I}_{2}(v-1)+\mathrm{He}
\end{aligned}
$$

Here, the $\mathrm{I}_{2}$ is excited to a high vibrational state $v$ in the excited electronic state. The results for the dependence of dissociation rate (assumed to be deduced from the linewidth) on $v$ have been interpreted principally in terms of radiationless transition theory (Beswick and Jortner). The system may well be one for which there is no energy "randomization," the only randomization being possible (before dissociation) being that between the bending vibration-rotational modes, the dissociation mode, but only one of the quanta of the $I_{2}$ vibration.

Other new techniques developed largely in the late 1970s and early 1980s to study intramolecular dynamics include the use of pumpprobe experiments, e.g., in the infrared multiphoton absorption of $\mathrm{SF}_{6}$ (Bolembergen et al.), of laser Raman excitation in the liquid phase (Kaiser and Laubereau), the one-quantum infrared excitation of methyl formate in one band, followed by luminescence from other vibrations (McDonald), dispersed fluorescence as compared with "discrete" fluorescence in aromatics, at relatively low vibrational excitation energies (Levy, Smalley, Parmenter, and their co-workers), and vibrational quantum beats (Zewail).

Recently, (1980s, largely) a new mode of excitation has been used-namely laser excitation of high $\mathrm{CH}$ overtones (e.g., Berry et al. and Crim et al.). Reactions such as isomerization of methyl isocyanide,

$$
\mathrm{CH}_{3} \mathrm{NC} \stackrel{h \nu \stackrel{\text { Cvertone }}{\text { ove }} k_{3}}{\longrightarrow} \mathrm{CH}_{3} \mathrm{CN}
$$

of allyl isocyanide, and (in real time) of the dissociation of tetramethyl dioxetane have been investigated. The $\mathrm{CH}_{3} \mathrm{NC}$ results could be compared with, and were found to be consistent with, those expected from RRKM theory. The allyl isocyanide data showed only a $50 \%$ fluctuation from a monotonic plot of $k_{3}(E)$ vs. $E$ curve $(E=$ energy 
of incident photon), and hence showed little, in my opinion, specificity to the vibrational mode excited.

In 1981-1983, Zewail and co-workers studied picosecond vibronic excitation of large molecules in jet beams, followed by dissociation or isomerization at low energies. The experimental results, described more fully by Zewail here and elsewhere and obtained at very low vibrational energies $\left(1000-5000 \mathrm{~cm}^{-1}\right)$ were compared with RRKM calculations. The unimolecular rates of cis-trans isomerization of stilbene and of bond breaking in hydrogen-bonded complexes were found to agree with RRKM calculations, given various current uncertainties in some of the parameters.

Very recently (1982) data have been obtained by Rowland et al. on the effect of a heavy metal atom or a chemical activation:

$$
\begin{aligned}
\mathrm{F}+\left(\mathrm{CH}_{2}=\mathrm{CH}-\mathrm{CH}_{2}\right)_{4} \mathrm{Sn} & \longrightarrow \mathrm{CH}_{2} \mathrm{CHFCH}_{2} \mathrm{Sn}\left(\mathrm{C}_{3} \mathrm{H}_{5}\right)_{3} \\
\mathrm{CH}_{2} \mathrm{CHFCH}_{2} \mathrm{Sn}\left(\mathrm{C}_{3} \mathrm{H}_{5}\right)_{3} & \stackrel{k_{3}}{\longrightarrow} \mathrm{CH}_{2}=\mathrm{CHF}+\text { radical } \\
\text { or } & \longrightarrow \text { collisional deactivation }
\end{aligned}
$$

The $k_{3}$ values, determined indirectly, were interpreted in terms of the $\mathrm{Sn}$ acting as a barrier to vibrational energy transfer between the initially vibrationally excited portion $\left(\mathrm{CH}_{2} \mathrm{CHFCH}_{2}\right)$ of the molecule and the rest of the molecule, i.e., the $k_{3}$ was closer to that expected for a small species than for the large species.

Equations for anharmonic vibrations in nonlinear mechanics have been extensively investigated in astronomy and other fields. In particular, energy and angular momentum (for spherical symmetry) or its $Z$-component (for cylindrical symmetry) are well-known constants of the motion. But the main questions have been (1) whether or not there are additional hidden constants of the motion which restrict the internal motion of the system (in our case, a molecule) in phase space, (2) what is the quantum mechanical counterpart of such a question, (3) what role does preparation of the state of the vibrationally excited molecule have on this question, and (4) what implications of these questions are there for the various experimental data.

We have recently reviewed some of these topics elsewhere and shall comment on them only relatively briefly here. The studies in nonlinear classical mechanics show that at low perturbations from "integrable" systems (e.g., low energies) these hidden constants of the motion, which severely restrict the motion in phase space, do 
exist for most initial conditions: the famous KAM theorem (Kolmogorov, Arnold and Moser) states that for small perturbations from an integrable system a system of $N$ coordinates moves, for most initial conditions, on an $N$-dimensional surface in phase space, rather than on a much larger $2 N-1$ (constant energy) surface, in $2 N$-dimensional phase space. This motion is variously termed "regular" or "quasiperiodic" in the literature. Under such conditions RRKM theory would not be applicable to individual classical "states." On the other hand, classical trajectory calculations show that at larger perturbations (e.g., higher energies in typical anharmonic systems) the motion frequently appears to be "chaotic." The primary assumption in N. B. Slater's theory is that the motion is regular (and, more particularly, simple harmonic), while one view of the assumptions of RRKM theory is that the motion is "chaotic." (In chaotic motion, neighboring classical trajectories separate exponentially rather than linearly with time, and so their ultimate fate is extremely sensitive to their initial conditions.)

Examples of regular and chaotic motion in a two coordinate system are given in Figures 1-2 and in Figure 3, respectively. Power spectra

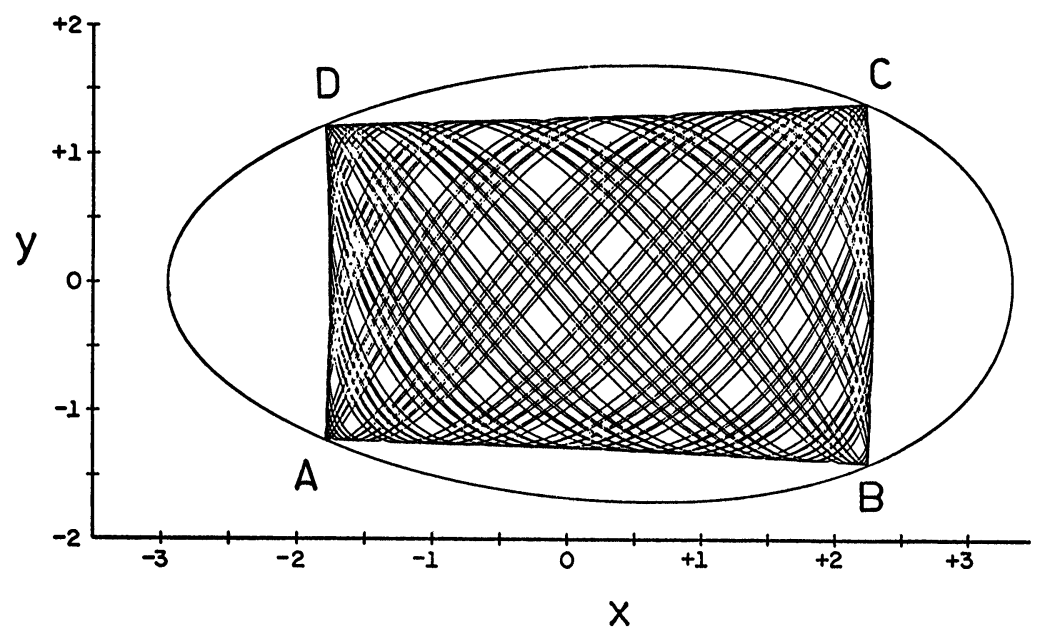

FIGURE 1 A quasi-periodic classical trajectory, $y(t)$ vs. $x(t)$ for the Hamiltonian $H=\frac{1}{2}\left(p_{x}^{2}+p_{y}^{2}+\omega_{x}^{2} x^{2}+\omega_{y}^{2} y^{2}\right)+\lambda x\left(x y+\eta x^{2}\right)$, with $\omega_{x}$ and $\omega_{y}$ incommensurate. The ellipse encloses the classically allowed region at the given total energy. At $A, B, C$ and $D, p_{x}=p_{y}=0$. One sees the effect of hidden constants of the motion which prevent the trajectory from filling up all of the space inside the ellipse. 


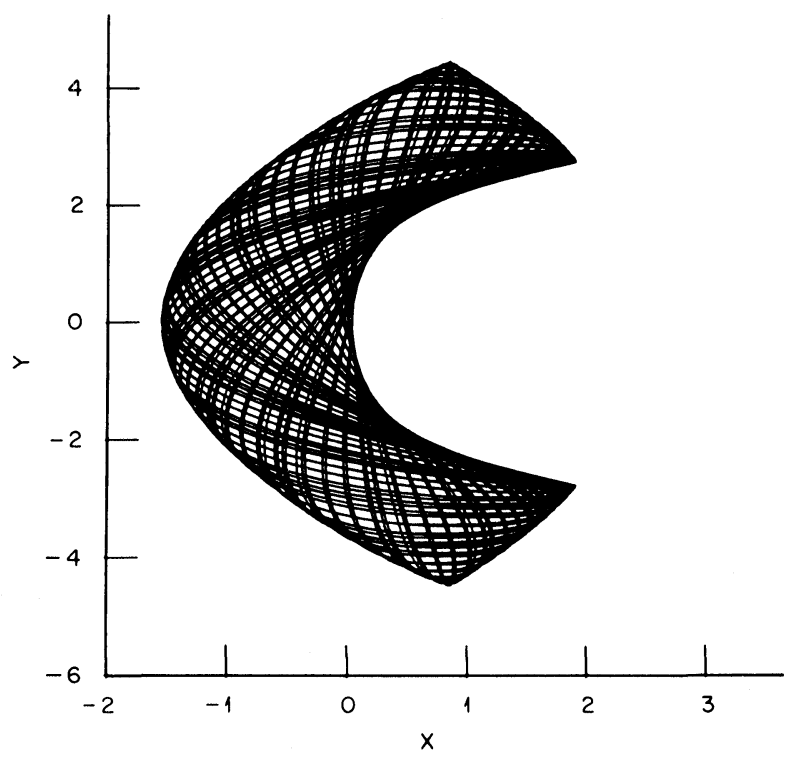

FIGURE 2 As in Figure 1, but with $\omega_{x}=2 \omega_{y}$.

calculated from autocorrelation functions from such trajectories are given in Figures 4 and 5, which correspond to the trajectories in Figures 1 and 3, respectively. The effect of the "chaotic" motion is to leave the principal vibration frequencies intact, but to "broaden" each vibrational absorption frequency.

Semiclassical arguments lead to the following quantum mechanical analog of this classical behavior: one expects that the vibrational spectrum of a molecule will be "regular" at low energies, perhaps corresponding to the "discrete" absorption at low energies in infrared multiphoton absorption. It is expected that when the classical motion is chaotic and when, simultaneously, the density of quantum states is high enough the spectrum will be "irregular" ("chaotic") at high energies. Perhaps this corresponds to the quasi-continuum at high energies in infrared multiphoton absorption and to the "dispersed" fluorescence observed in vibronic excitation. This dispersed fluorescence has usually been taken as a reflection of some extensive anharmonic mixing of the zeroth order modes of vibration.

The detailed connection between classical and quantum behavior is quite well understood in the case of quasiperiodic classical motion. 


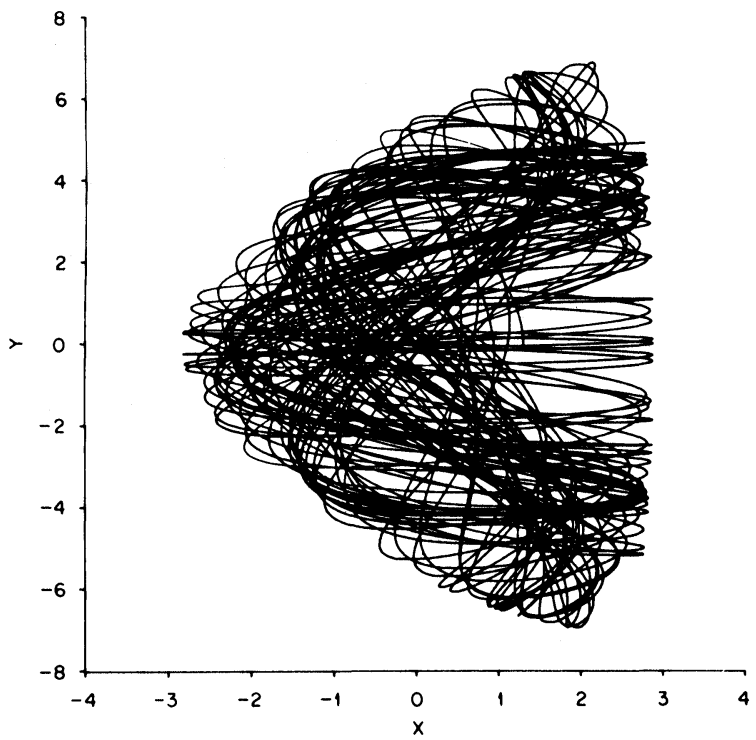

FIGURE 3 A chaotic trajectory for the Hamiltonian in Figure 1, but with $\omega_{x}=2 \omega_{y}$. (The energy is higher than that in Figure 2.)

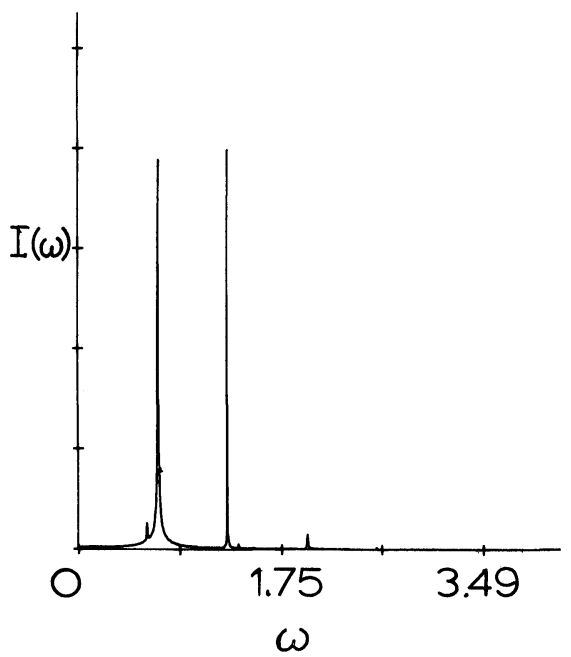

FIGURE 4 Power spectrum for the variable $x+y$ for a trajectory similar to that in Figure 1. 


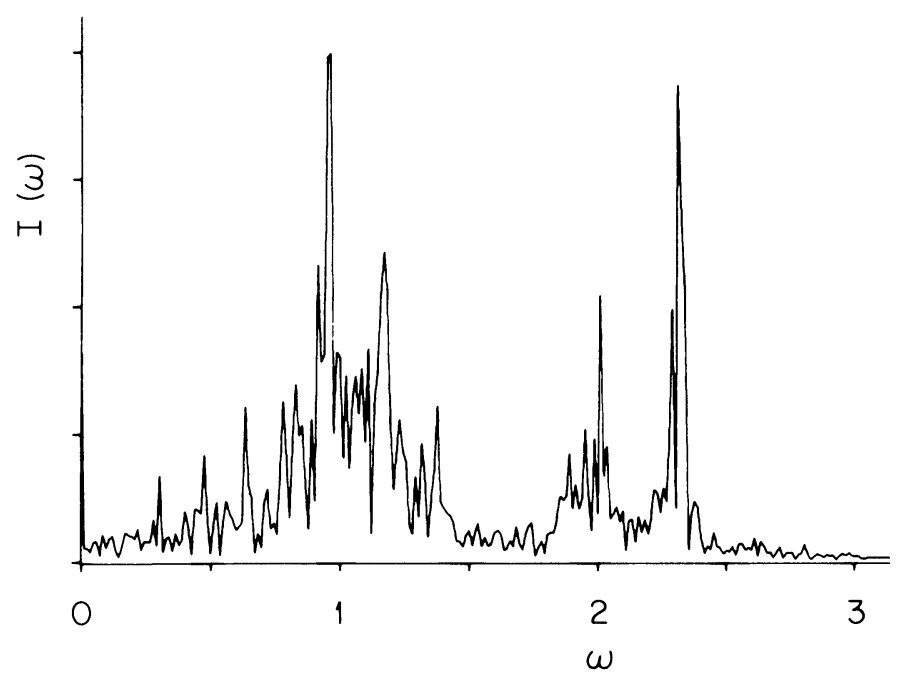

FIGURE 5 Power spectrum for the variable $x+y$ for the trajectory of Figure 3 .

Wavefunctions of "regular" states show regular nodal patterns. (E.g., Figure 6 and compare with Figure 2.) A suitable semiclassical theory is available, formulated on the basis of arguments by Einstein, Brillouin and Keller. The first practical evaluation of the "phase integrals" (needed for semiclassical quantization of classical trajectories), for nonseparable anharmonic systems with smooth potentials, was given by Eastes (1974) and Noid (1975) in our group.

The connection between classical and quantum behavior in the case of chaotic classical states is the subject of current research by a number of theoretical groups. For example, "classical chaos" is not expected to imply "quantum chaos" if the density of quantum states is too low. We have discussed elsewhere this latter connection in terms of "overlapping avoided crossings" plots of eigenvalues. We have also noted that extensive anharmonic mixing of zeroth order states can occur either "quasiperiodically" or chaotically. In the former case, there continue to be regular wave patterns, though quite different from those of the zeroth order states. Such mixing affects Franck-Condon factors, for example, though in somewhat different ways in the two cases. In the chaotic case the wavefunction is "bumpy" rather than exhibiting the regular nodal patterns. 


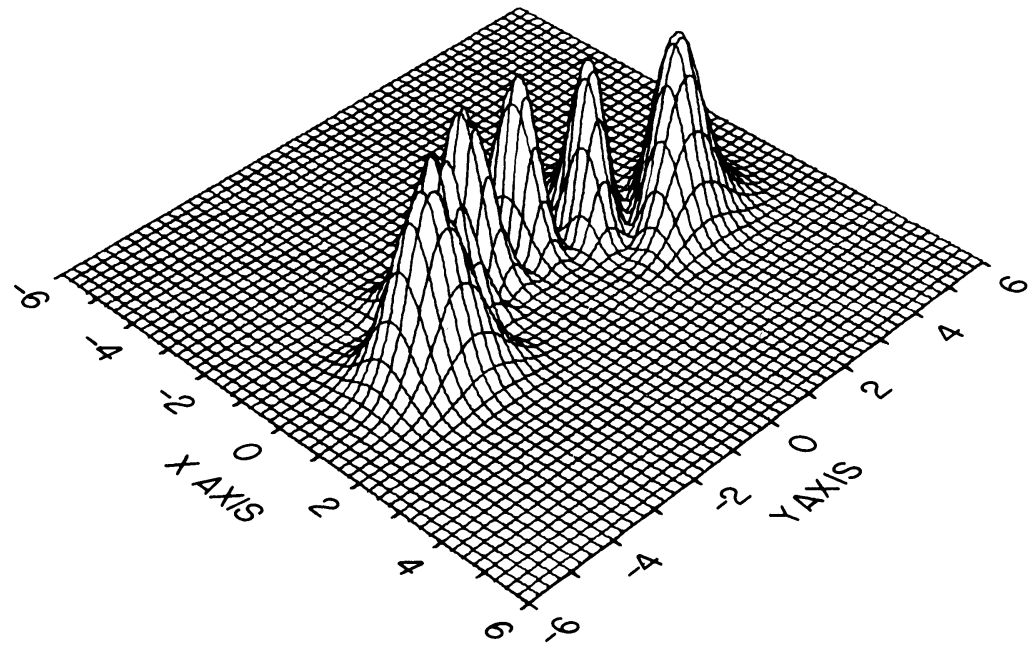

FIGURE 6 A quantum mechanical wavefunction $\left(|\psi|^{2}\right)$ which corresponds semiclassically to the trajectory in Figure 2 . Note that the region of coordinate space "supporting" this wavefunction is the same as the region occupied by the corresponding classical trajectory ("eigentrajectory") in Figure 2.

The preparation of the state of the system and its relation to ensuing observations have been extensively discussed for radiationless transitions-such as the lifetime behavior, initial dephasing of the packet of excited states, exponential versus nonexponential decay, and other aspects. In the case of unimolecular processes, be they induced by collisions, by chemical activation, or by $\mathrm{CH}$ overtone excitation, a wave packet of states is again excited.

A question of particular interest is whether or not during the time evolution of the decomposing molecule this wavepacket explores all of the available "quantum states" within the energy width of the wavepacket, so that Eq. (5) is fulfilled for the rate constant, or whether it at least explores a sufficient representative number of "states" in the region of the transition state and of the parent molecule, such that the ratio $N^{+} / \rho$ in Eq. (5) is still approximately fulfilled. For example, if there are many "overlapping avoided crossings" as a result of extensive anharmonic mixing (discussed elsewhere), the vibrational wave functions of the various quasi-bound states should have a 
"statistical" character with respect to the nodal patterns. In this way the spatial and dynamical behavior of a small number of "statistical" states in a wave packet might provide a type of "coarse graining" of the behavior in a microcanonical distribution.

The lifetime behavior of the vibrationally-excited molecule, reflected in the many types of studies of unimolecular reactions described earlier, is consistent with an extensive anharmonic mixing of the various zeroth-order states constituting the wave packet. To provide a detailed description of the individual states, however, would require an analysis of a sufficiently high resolution spectra on the same systems, a formidable task.

We turn next to the possibility of an intramolecular laser selective chemistry. This goal has been an elusive one. Recently, we made a suggestion, stimulated, in part, by the results of Rowland et al. on reactions (19)-(21) which we described earlier and, in part, by following calculations by Lopez in our group.

In classical trajectory calculations, we found that the extent of energy transfer across $\mathrm{Sn}$ in a linear CCCSnCCC chain of Morse oscillators was small when the energy of the initially excited CCC was large, but was relatively extensive (on a percentage basis) when this excess energy of the initially excited CCC was small. The effect disappeared when the Morse oscillators were replaced by harmonic ones or when the mass of the central atom was sufficiently decreased. The angle between the two ligands is also an important factor, since it affects the "effective mass" in the $(\mathrm{C}-\mathrm{Sn}, \mathrm{Sn}-\mathrm{C})$ bond moment coupling term in the kinetic energy, a tetrahedral angle corresponding to an increase in effective mass of a factor of four over that for a $180^{\circ}$ bond angle.

All of these effects can be explained in terms of resonance or off-resonance between the vibration of the two ligands. A high vibrational energy in one CCC causes an anharmonic red shift of the frequencies, and makes for an off-resonance behavior, when the low energy behavior was on-resonance, as in the case CCCSnCCC.

A possible way of exploiting such results, if applicable to real molecules, to obtain an intramolecular laser-selective chemistry, involves the preparation of a molecular in which different reactions can occur in the different ligands:

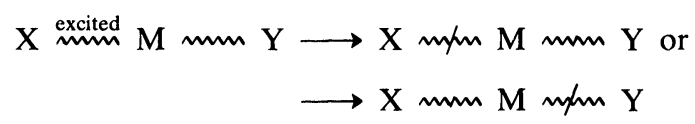


where the wiggly lines denote carbon or other chains and the dash indicates bond rupture or isomerization. The synthesis of such molecules is being explored at Caltech. The excitation can be by $\mathrm{CH}$ or $\mathrm{OH}$ overtones, by infrared multiphoton absorption, or by chemical activation.

It is perhaps clear from the present summary that the field of unimolecular reactions has become a many-faceted one during the past sixty years. The wide variety of kinetic and spectroscopic techniques now being developed, and the many current studies of anharmonic behavior, serve to define more sharply some of the questions which have been raised. The next decade promises to be an exciting one in this field.

\section{Acknowledgements}

It is a pleasure to acknowledge the support of this research by the National Science Foundation. This is contribution No. 6805, California Institute of Technology.

\section{References}

Some of the history prior to the 1970 s is described in several volumes, e.g., Refs. 1-5. More recent reviews of new experiments ${ }^{6-14}$ and of various theoretical anharmonic developments ${ }^{14-16}$ have been given and contain many references to other reviews and articles. In the interests of brevity we refer the reader to these articles and to still more recent results in journals such as J. Chem. Phys. and Chem. Phys. Lett.

1. P. L. Robinson and K. A. Holbrook, Unimolecular Reactions (Wiley-Interscience, New York, 1972).

2. W. Forst, Unimolecular Reactions (Academic Press, New York, 1973).

3. S. W. Benson, The Foundations of Chemical Kinetics (McGraw-Hill, New York, 1960).

4. K. J. Laidler, Theories of Chemical Reaction Rates (McGraw-Hill, New York, 1969).

5. E. E. Nikitin, Thermally Induced Gas Phase Reactions (Indiana University Press, Bloomington, 1966).

6. D. H. Levy, Annu. Rev. Phys. Chem. 31, 197 (1980).

7. J. D. McDonald, Annu. Rev. Phys. Chem. 30, 29 (1979).

8. I. Oref and B. S. Rabinovitch, Acc. Chem. Res. 12, 166 (1969).

9. P. A. Schulz, Aa. Sudbø, D. J. Krajnovich, H. S. Kwok, Y. R. Shen and Y. T. Lee, Annu. Rev. Phys. Chem. 30, 379 (1979).

10. J. B. Hopkins, D. W. Powers and R. E. Smalley, J. Chem. Phys. 73, 683 (1980).

11. K. V. Reddy and M. J. Berry, Faraday Disc. Chem. Soc. 67, 188 (1979).

12. B. D. Cannon and F. F. Crim, J. Chem. Phys. 73, 3013 (1980).

13. A. H. Zewail, this conference.

14. D. W. Noid, M. L. Koszykowski and R. A. Marcus, Annu. Rev. Phys. Chem. 32, 267 (1981).

15. I. C. Percival, Adv. Chem. Phys. 36, 1 (1977).

16. R. A. Marcus, Faraday Disc. Chem. Soc. 75, (1983). 